\title{
Single Photon Emission Computed Tomography of Technetium-99m Tetrofosmin Myocardial Perfusion Imaging in Patients with Systemic Lupus Erythematosus-A Preliminary Report
}

\author{
Jen-Jhy LIN, ${ }^{1}$ MD, Hsiu-Bao Hsu, ${ }^{1}$ MD, Shung-Shung Sun, ${ }^{2}$ MD, \\ Jhi-Joung WANG ${ }^{3} \mathrm{MD}$, Shung-Tai $\mathrm{HO},{ }^{4} \mathrm{MD}$, and Chia-Hung $\mathrm{KAO},{ }^{2} \mathrm{MD}$
}

\section{SUMMARY}

The purpose of this study was to evaluate the utility of single-photon emission computed tomography (SPECT) of technetium-99m tetrofosmin (Tc-99m TF) myocardial perfusion imaging to detect myocardial involvement in patients with systemic lupus erythematosus (SLE).

Three groups of subjects-group 1: 25 SLE female patients with non-specific cardiac symptoms and signs, group 2: 25 female SLE patients without any cardiac symptoms and signs, and group 3: 25 female healthy controls-were evaluated by comparing rest and dipyridamole-stress Tc-99m TF myocardial perfusion SPECT.

Tc-99m TF myocardial perfusion SPECT revealed perfusion defects in $88 \%$ and $40 \%$ of the cases in groups 1 and 2, respectively. However, no cases in group 3 demonstrated myocardial perfusion defects.

Tc-99m TF myocardial perfusion SPECT is a useful noninvasive imaging modality to detect cardiac involvement in SLE patients with or without cardiac symptoms and signs. (Jpn Heart J 2003; 44: 83-89)

Key words: Technetium-99m tetrofosmin, Single photon emission computed tomography, Systemic lupus erythematosus

AUTOPSY studies have documented that the heart is affected in most patients with systemic lupus erythematosus (SLE), although clinical manifestations only occur in less than $10 \%{ }^{1,2)}$ However, the diagnosis of cardiac involvement in SLE is difficult because of the lack of useful noninvasive imaging methods to detect cardiac involvement and clinical cardiac manifestations are only found in a few SLE patients.

From ${ }^{1}$ Division of Cardiology and ${ }^{2}$ Department of Nuclear Medicine, China Medical College Hospital, Taichung,

${ }^{3}$ Department of Medical Research, Chi-Mei Medical Center, Tainan, ${ }^{4}$ School of Medicine, National Defense Medical Center, Taipei, Taiwan.

Address for correspondence: Chia-Hung Kao, MD, Department of Nuclear Medicine, China Medical College Hospital, No.

2, Yuh-Der Road, Taichung 404, Taiwan.

Received for publication November 19, 2001.

Revised and accepted June 28, 2002. 
Thus, it is necessary to use a noninvasive method for the early detection of subclinical cardiac involvement in SLE patients to prevent later cardiac events. Technetium-99m (Tc-99m) sestamibi myocardial perfusion single photon emission computed tomography (SPECT) has been used in the evaluation of myocardial perfusion defects in asymptomatic lupus patients. ${ }^{3,4)}$ Tc-99m tetrofosmin, competing with Tc-99m sestamibi, has been used as a newer radiopharmaceutical for myocardial imaging and is considered to have mechanisms similar to those of Tc-99m sestamibi. ${ }^{5,6)}$ In addition, Tc-99m tetrofosmin is more easily prepared without boiling and achieves more optimal biodistribution for myocardial imaging than Tc-99m sestamibi. ${ }^{7)}$

Therefore, the objective of the present study was to assess the utility of Tc$99 \mathrm{~m}$ tetrofosmin myocardial perfusion SPECT in the detection of cardiac involvement in SLE patients with or without symptoms and signs of heart disease.

\section{Materials ANd Methods}

Patients: Two groups of female patients with a definite diagnosis of SLE according to the revised criteria of the American College of Rheumatology (9) were enrolled in this study. Group 1 included 25 patients (34.5 \pm 7.4 years old) with nonspecific cardiac symptoms and signs (chest discomfort and/or dyspnea and/or occasional palpitations). Group 2 included 25 SLE patients (33.8 \pm 7.7 years old) without any cardiac symptoms and signs. In addition, a third group (group 3) of 25 female healthy subjects (33.8 \pm 7.5 years old) was also included as controls. None of the SLE patients had any involvement of major organs such as the brains, lungs, and kidneys. None of the study subjects in any group had a previous history of heart disease such as pericarditis or myocarditis, cardiac risk factors such as hypertension or hyperlipidemia, abnormal chest X-ray findings, or abnormal resting EKG findings. Each subject underwent Tc-99m tetrofosmin myocardial perfusion SPECT to detect myocardial perfusion abnormalities. Serum levels of anticardiolipin antibodies (aCL) were measured using enzyme linked immunoassay kits (Quanta Lite ${ }^{\mathrm{TM}}$ ACA IgG/IgM and IgA, INOVA Diagnostics, Inc., San Diego, CA, USA).

Rest and dipyridamole-stress technetium-99m tetrofosmin myocardial perfusion single photon emission computed tomography: We were concerned that some drugs for treating SLE may influence the results of myocardial perfusion SPECT, therefore, all treatment was withheld for 24 hours before Tc-99m TF myocardial perfusion SPECT. All patients joined the study after at least a 4-hour fast and had refrained from ingesting products containing methylxanthines for at least 24 hours before the test. We used the one-day rest-stress protocol. Rest SPECT 
imaging was undertaken 1 hour after intravenous injection of 7.4-11.1 MBq of Tc- $99 \mathrm{~m} \mathrm{TF} / \mathrm{kg}$. Thereafter, dipyridamole was administered intravenously at a rate of $0.56 \mathrm{mg} / \mathrm{kg}$ for 4 minutes. Three minutes after dipyridamole infusion, another higher dose of Tc-99m TF (3 times the rest dose) was administered. Intravenous aminophylline $(3-5 \mathrm{mg} / \mathrm{kg}$ ) was administered as needed in the recovery period (at least 3 minutes after Tc-TF injection). Stress SPECT imaging was undertaken 1 hour later. SPECT images were acquired using a large field of view dual-headed gamma camera (ADAC, Vertex plus) equipped with a low-energy, all purpose, parallel-hole collimator. Data were obtained from 64 projections of 25 seconds each in the $140 \mathrm{keV}$ photopeak over a $180^{\circ}$ arc in a $64 \times 64$ matrix. Short-axis, vertical long-axis and horizontal long-axis were reconstructed from the raw data by filtered backprojection with a Butterworth filter with a cutoff frequency of 0.5 and an order of 10 in the rest and cutoff frequency of 0.66 and an order of 5 in the stress studies. Regions of interest were defined as apical; and anterior, lateral and inferior free wall. A perfusion defect was defined as when less than $50 \%$ of the surrounding myocardium (including moderate and severe perfusion defects) was observed. All defects were graded as a fixed defect (FD, a defect that was present both in the rest and stress SPECT images), (Figure 1) a transient defect (TD, a defect that was present only in the stress SPECT image), (Figure 2) and a reverse

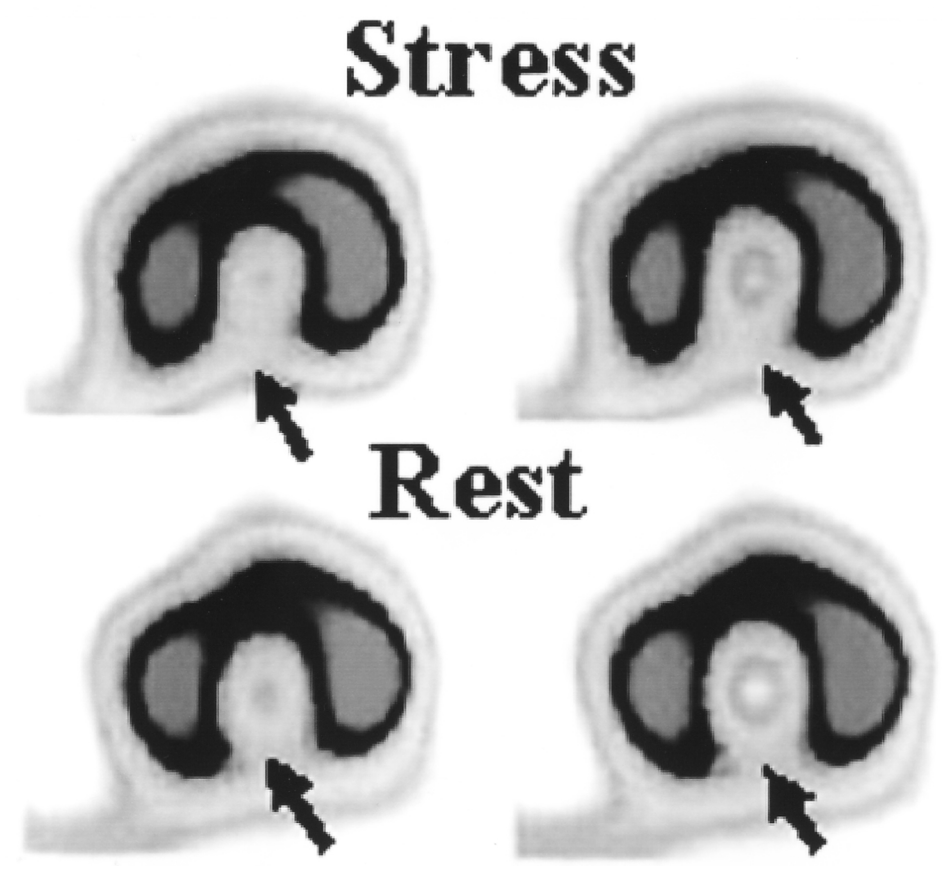

Figure 1. Rest and dipyridamole-stress Tc-99m TF myocardial perfusion SPECT revealed a fixed defect in the inferior wall (arrows). 
defect (RD, a defect that was present only in the rest SPECT image), (Figure 3)

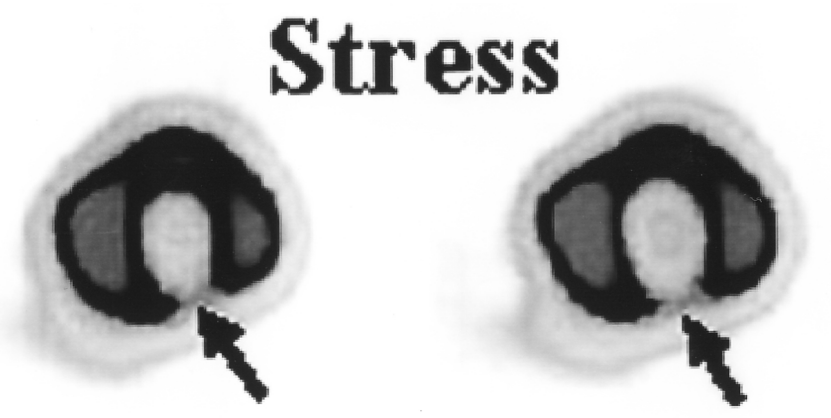

\section{Rest}
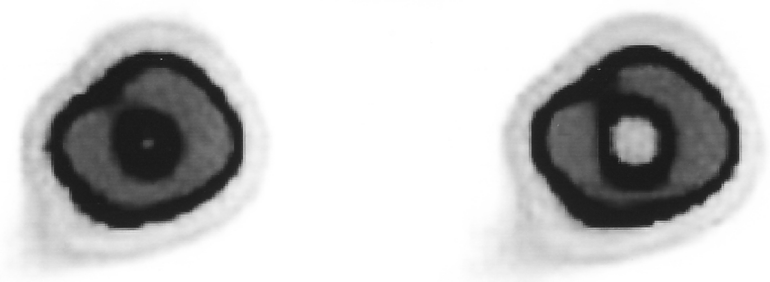

Figure 2. Rest and dipyridamole-stress Tc-99m TF myocardial perfusion SPECT revealed a transient defect in the inferior wall (arrows).

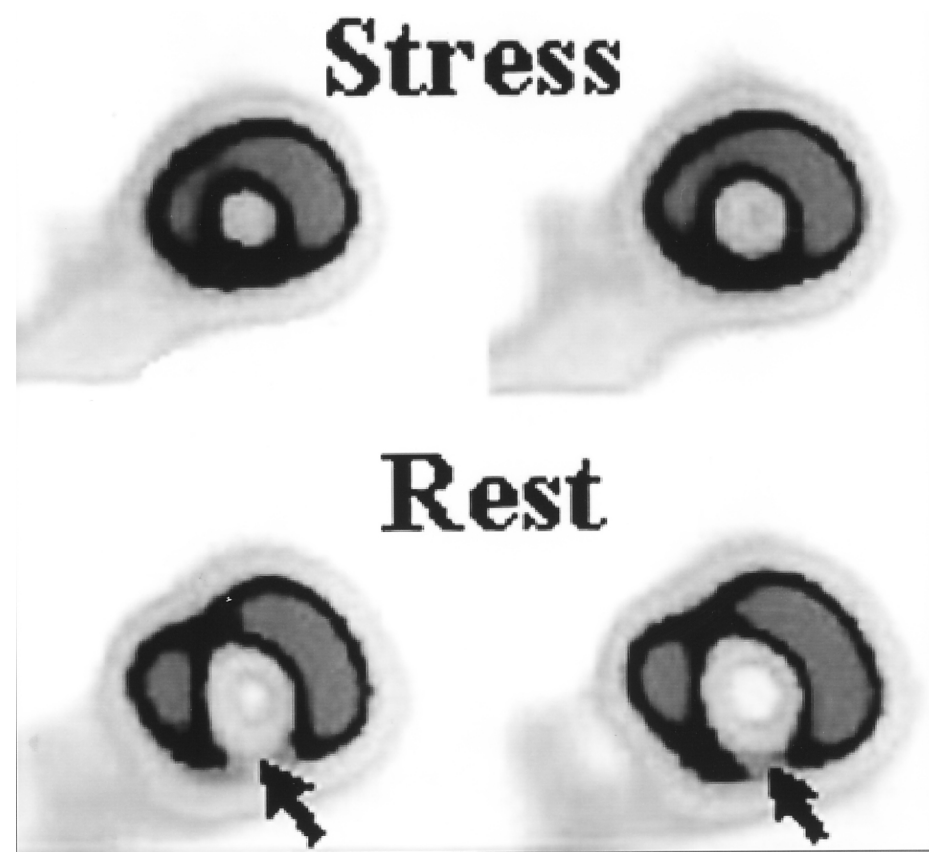

Figure 3. Rest and dipyridamole-stress Tc-99m TF myocardial perfusion SPECT revealed a reverse defect in the inferior wall (arrows). 
All SPECT images were interpreted by a consensus of 2 experienced observers, with a third interpreting in cases of a disagreement.

\section{RESULTS}

Detailed results and the data for patients in groups 1 and 2 are shown in Table I. No significant adverse reaction occurred in any patient after dipyridamole infusion. A disagreement in the interpretation of results of Tc-99m TF myocardial perfusion SPECT that needed a third interpretation only occurred in 2 cases. No definite perfusion defect was found in any of the group 3 healthy controls. Myocardial perfusion defects were noted in $22 / 25(88 \%)$ of group 1

Table I. Detailed Data for Group 1 and Group 2 Patients

\begin{tabular}{|c|c|c|c|c|c|c|c|c|c|c|}
\hline \multicolumn{6}{|c|}{ Group 1} & \multicolumn{5}{|c|}{ Group 2} \\
\hline \multirow{2}{*}{$\begin{array}{l}\text { Case } \\
\text { No. }\end{array}$} & \multirow{2}{*}{$\begin{array}{l}\text { Age } \\
\text { (years) }\end{array}$} & \multicolumn{3}{|c|}{$\begin{array}{l}\text { Tc-99m TF Myocardial Perfusion } \\
\text { SPECT }\end{array}$} & \multirow[b]{2}{*}{$\mathrm{aCL}$} & \multirow{2}{*}{$\begin{array}{c}\text { Age } \\
\text { (years) }\end{array}$} & \multicolumn{4}{|c|}{$\begin{array}{l}\text { Tc-99m TF Myocardial } \\
\text { Perfusion SPECT }\end{array}$} \\
\hline & & FD & TD & $\mathrm{RD}$ & & & FD & TD & $\mathrm{RD}$ & $\mathrm{aCL}$ \\
\hline 1 & 21 & - & Ant,Inf & - & - & 20 & - & - & - & - \\
\hline 2 & 22 & Ant,Sep & - & - & - & 21 & Ant & - & - & + \\
\hline 3 & 24 & Inf & Ant & - & - & 23 & - & - & - & + \\
\hline 4 & 25 & - & Ant & - & - & 24 & - & - & - & - \\
\hline 5 & 26 & Ant & - & - & + & 25 & - & Inf & - & + \\
\hline 6 & 27 & - & Ant,Inf & - & + & 25 & $\operatorname{Inf}$ & - & - & - \\
\hline 7 & 30 & - & Inf & - & - & 28 & - & - & - & + \\
\hline 8 & 31 & - & Inf & - & + & 29 & Ant,Sep & - & - & + \\
\hline 9 & 33 & - & Ant,Inf & - & + & 31 & - & - & - & - \\
\hline 10 & 33 & - & Ant,Inf & - & - & 32 & - & - & - & - \\
\hline 11 & 34 & - & - & $\operatorname{Inf}$ & + & 34 & - & $\operatorname{Inf}$ & - & + \\
\hline 12 & 34 & Inf & - & - & - & 34 & - & - & - & - \\
\hline 13 & 34 & - & - & - & + & 35 & - & - & - & + \\
\hline 14 & 35 & - & - & - & + & 35 & - & - & Inf & - \\
\hline 15 & 36 & - & Ant,Inf,Apex & - & + & 37 & - & - & - & - \\
\hline 16 & 37 & - & Ant,Sep,Inf & - & - & 38 & - & Ant & - & + \\
\hline 17 & 38 & - & Ant & - & + & 38 & - & - & - & - \\
\hline 18 & 39 & Ant & - & - & + & 39 & - & - & - & + \\
\hline 19 & 41 & - & Ant,Inf & - & - & 40 & Ant & - & - & - \\
\hline 20 & 42 & - & $\operatorname{Inf}$ & - & - & 41 & Ant & Sep & - & - \\
\hline 21 & 42 & - & Inf & Ant & - & 41 & - & - & - & + \\
\hline 22 & 44 & - & Ant & $\operatorname{Inf}$ & + & 42 & - & - & - & + \\
\hline 23 & 44 & Ant,Inf,Apex & - & - & - & 43 & - & - & - & - \\
\hline 24 & 45 & - & - & - & + & 45 & - & - & - & - \\
\hline 25 & 46 & - & Ant & - & - & 46 & - & Ant, Inf & - & - \\
\hline
\end{tabular}

$\mathrm{FD}=$ fixed defects; $\mathrm{TD}=$ transient defects; $\mathrm{RD}=$ =reverse defects; Ant=anterior segment; Inf=inferior segment; Sep=Septal segment; -=negative finding; $\mathrm{aCL}=$ anticardiolipin antibodies. 
patients: 6 patients had FD, 16 patients had TD, and 3 patients had RD (1 patient with both FD and TD, 2 patients with both TD and RD). Myocardial perfusion defects were noted in 10/25 (40\%) group 2 patients: 5 patients had FD, 5 patients had TD, and 1 patient had RD. The incidence of myocardial perfusion defects in group 1 was significantly higher than that in group $2(P$ value $<0.01$, Fisher exact test). Minor positive changes of slight ST-depression $(<1 \mathrm{~mm})$ in 2 were observed in the EKG symptomatic SLE patients (group 1) and in 1 asymptomatic SLE patient (group 2). Six patients in group 1 and 2 patients in group 2 experienced some discomfort during the dipyridamole stress test (dizziness or headache or flushing or chest pain); however, all of the symptoms and signs were mild, well tolerated and promptly reversed by aminophylline. No EKG changes or chest discomfort symptoms and signs were found after dipyridamole infusion in the healthy female subjects (group 3). Data on aCL were also obtained in all of the SLE patients. However, no definite correlation was found between the results of Tc-99m TF myocardial perfusion SPECT and the clinical features and aCL data.

\section{DiSCUSSION}

The clinical manifestations seen in SLE patients related to heart diseases (pericarditis, myocarditis, valvular disease, coronary artery disease, etc) such as chest discomfort, dyspnea and occasionally palpitations are nonspecific clinical symptoms and signs. ${ }^{8}$ Thus, the symptoms and signs of group 1 patients in this study were nonspecific. Coronary artery disease (CAD) has been recognized as a major cause of morbidity in patients with SLE and the cardiac involvement is now considered to be the third leading cause of death in SLE. ${ }^{8-11)}$ The frequency of clinically recognizable CAD in SLE was reported to be between $6.1 \%$ and $8.9 \%,{ }^{12,13)}$ however, previous studies have reported that the frequency of subclinical myocardial perfusion defects in patients with SLE is higher., ${ }^{3,4)}$ It is not surprising that a higher prevalence of myocardial perfusion defects shown by rest and dipyridamole-stress Tc-99m TF myocardial SPECT was found in $88 \%$ of the group 1 patients with nonspecific cardiac symptoms and signs, because a high prevalence $(40 \%)$ was also noted in the group 2 patients without cardiac symptoms and signs and our findings were similar to the results of a previous study ${ }^{4}$ using Tc-99m sestamibi myocardial perfusion imaging.

In our series, none of the patients was submitted to coronary angiography. Therefore, in this study, we do not think the patterns and location of the defects in Tc-99m TF myocardial perfusion SPECT will be helpful for differentiating between large- and small-vessels disease. However, Schillaci, et $a l^{3)}$ reported normal coronary angiography results in all SLE patients with a positive Tc-99m sestamibi myocardial SPECT scan. These findings suggest intramyocardial small 
vessel disease due to the primary immunological damage of SLE. After reviewing the literature, we found only one autoantibody (aCL) was possibly related to cardiac involvement in SLE patients. ${ }^{14)}$ However, we found no significant correlation between the aCL data and the SPECT results in our study.

In conclusion, our preliminary results have showed that Tc-99m tetrofosmin myocardial perfusion SPECT is a useful noninvasive imaging method for the early detection of cardiac involvement in SLE patients with nonspecific symptoms/signs. However, further follow-up studies with larger study populations are needed to evaluate whether patients with these myocardial perfusion defects are at higher risk of future cardiac events.

\section{REFERENCES}

1. Mandell BF. Cardiovascular involvement in systemic lupus erythematosus. Semin Arthritis Rheum 1987; 17: 126-41.

2. Estes D, Christian CL. The natural history of systemic lupus erythematosus by prospective analysis. Medicine 1971; 50: 85-95.

3. Schillaci O, Lagana B, Danieli R, et al. Technetium-99m sestamibi single-photon emission tomography detects subclinical myocardial perfusion abnormalities in patients with systemic lupus erythematosus. Eur J Nucl Med 1999; 26: 713-7.

4. Hosenpud JD, Montanaro A, Hart MV, et al. Myocardial perfusion abnormalities in asymptomatic patients with systemic lupus erythematosus. Am J Med 1984; 77: 286-92.

5. Higley B, Smith FW, Smith T, et al. Technetium-99m-1, 2-bis (bis (2-ethoxyethyl) phosphino) ethane: human biodistribution, dosimetry and safety of a new myocardial perfusion imaging agent. J Nucl Med 1993; 34: 30-8.

6. Kelly JD, Forster AM, Higley B, et al. Technetium-99m-tetrofosmin as a new radiopharmaceutical for myocardial perfusion imaging. J Nucl Med 1993; 34: 222-7.

7. Flamen P, Bossuyt A, Franken PR. Technetium-99m-tetrofosmin in dipyridamole- stress myocardial SPECT imaging: intraindividual comparison with technetium-99m-sestamibi. J Nucl Med 1995; 36: 2009-15.

8. Doherty NE, Siegel RJ. Cardiovascular manifestations of systemic lupus erythematosus. Am Heart J 1985; 110: $1257-65$.

9. Rubin LA, Urowitz MB, Gladman DD. Mortality in systemic lupus erythematosus: the bimodal pattern revisited. Q J Med 1985; 55: 87-98.

10. Wallace DJ, Podell T, Weiner J, Klinenberg JR, Forouzesh S, Dubois EL. Systemic lupus erythematosus--survival patterns. Experience with 609 patients. JAMA 1981; 245: 934-8.

11. Gladman DD, Urowitz MB. Morbidity in systemic lupus erythematosus. J Rheumatol 1987; 14: 223-6.

12. Petri M, Perez-Gutthann S, Spence D, Hochberg MC. Risk factors for coronary artery disease in patients with systemic lupus erythematosus. Am J Med 1992; 93: 513-9.

13. Shome GP, Sakauchi M, Yamane K, Takemura H, Kashiwagi H. Ischemic heart disease in systemic lupus erythematosus. A retrospective study of 65 patients treated with prednisolone. Jpn J Med 1989; 28: 599-603.

14. Sturfelt G, Eskilsson J, Nived O, Truedsson L, Valind S. Cardiovascular disease in systemic lupus erythematosus-a study of 75 patients from a defined population. Medicine 1992; 71: 216-23. 